\title{
Hospital readmissions at a tertiary care children's hospital: Prevalence, associated characteristics, and preventability
}

\author{
Daiana Basso, M.D. ${ }^{a}$, Carolina Bermúdez, M.D. ${ }^{a}$, Sofía Santoro Gallardo, M.D. ${ }^{a}$, \\ Francisco Tonini, M.D. ${ }^{a}$, Federico Torres, M.D. ${ }^{a}$, Fernando Ferrero, M.D. ${ }^{a}$ and \\ Mariano Ibarra, M.D. ${ }^{a}$
} ABSTRACT
Introduction. Hospital readmissionsin pediatrics
are a severe, potentially avoidable problem
of health systems. In our setting, there is little
information about this topic.
Objective. To estimate the rate of readmissions,
the proportion of potentially preventable
readmissions, and theirassociated characteristics.
Material and methods. Cross-sectional study
including hospital readmissions of patients aged
$0-18$ years, admitted to a tertiary care children's
hospital between January 1 st and December 31 st,
2018. Readmissions were assessed as potentially
preventable based on whether they were or not
related to the previous admission.
Results. Outof 8228 hospital admissions recorded
in the study period, the rate of readmissions for
any cause was $10 \%$ at 30 days and $7.1 \%$ at 15 days.
The proportion of readmissions classified as
potentially preventable was $47.9 \%$ at 30 days
and $47.5 \%$ at 15 days. No statistically significant
differences were observed between readmissions
at 30 and 15 days in terms of patient age, health
insurance, presence of chronic disease or cause
of readmission.
Conclusion. The rate of hospital readmissions
was $10 \%$ at 30 days and $7.1 \%$ at 15 days of
discharge; almost half of them were considered
potentially preventable.
Key words: patient readmission, health care quality,
child child.

http: / / dx.doi.org/10.5546/aap.2021.eng.e435

a. Hospital General de Niños Pedro de Elizalde, Autonomous City of Buenos Aires, Argentina.

E-mail address:

Mariano Ibarra, M.D.: marianoestebanibarra@ gmail.com

Funding:

None.

Conflict of interest:

None.

Received: 3-14-2021

Accepted: 4-28-2021

\section{INTRODUCTION}

Hospital readmissions (HR) are a severe problem of health systems. Since the implementation of Medicare's Hospital Readmissions Reduction Program in the United States, the rate of readmissions for any cause at 30 days has served as a measure of health care quality still at the heart of the debate. Although that program is a Medicare initiative targeted at adults, the interest in pediatric readmissions is now rising because they are a challenge for health systems worldwide. ${ }^{1,2}$ Pediatric HR are a burden on patients, families, and health systems. ${ }^{3}$

In spite of the growing interest on this topic, the reliability of readmission metrics as a health care quality measurement is at stake. Concerns are based on the following: a) great variations in the rate of readmission between different hospitals and the effect of socioeconomic factors on such variations, ${ }^{4}$ b) difficulty to assess whether readmissions are preventable, ${ }^{5}$ and c) the fact that, in spite of the efforts made to reduce the rate of readmissions, it remains unchanged. ${ }^{6}$

This subject is clearly installed in the United States, but there is little information about pediatric readmissions in South America. In Argentina, particularly, readmissions are not part of health care quality indicators, so there is no health policy in place in this regard.

Since pediatric readmissions have specific characteristics in each health system, and considering the lack of local information, this study explores the rate of readmissions, 
the proportion of potentially preventable readmissions, and their associated characteristics in a tertiary care children's hospital located in the Autonomous City of Buenos Aires.

\section{POPULATION, MATERIALS AND METHOD}

Design: Cross-sectional study.

Inclusion criteria: Hospital admissions of patients aged 0-18 years, admitted to a tertiary care children's hospital between January $1^{\text {st }}$ and December 31'st 2018.

Exclusion criteria: Admissions for which medical records did not include the data necessary for the study.

Primary outcome variable: HR were considered based on 2 periods: at 15 and 30 days after discharge. In addition, readmissions were categorized into "for any cause" and "for a potentially preventable cause". Potentially preventable readmissions were those not scheduled and related to the previous admission because they were: a) a recurrence of the same reason for admission, b) an acute decompensation of a chronic condition that may or may have not been the reason for the previous admission, or c) a complication related to the previous admission (criteria modified from Goldfield et al. ${ }^{7}$ ).

This way, outcome variables were as follows:

- HR for any cause in the 30-day period.

- HR for any cause in the 15-day period.

- HR for a potentially preventable cause in the 30-day period.

- HR for a potentially preventable cause in the 15-day period.

Other variables: Characteristics of patients and their hospitalization, including age, exclusively public health insurance, presence of an underlying chronic condition, month, and length of stay (days) at readmission.

Study procedure: It was carried out in 2 stages (Figure 1). For the first stage, data from all patients admitted during the study period were obtained from the Admission and Discharge Office in order to estimate the prevalence of HR for any

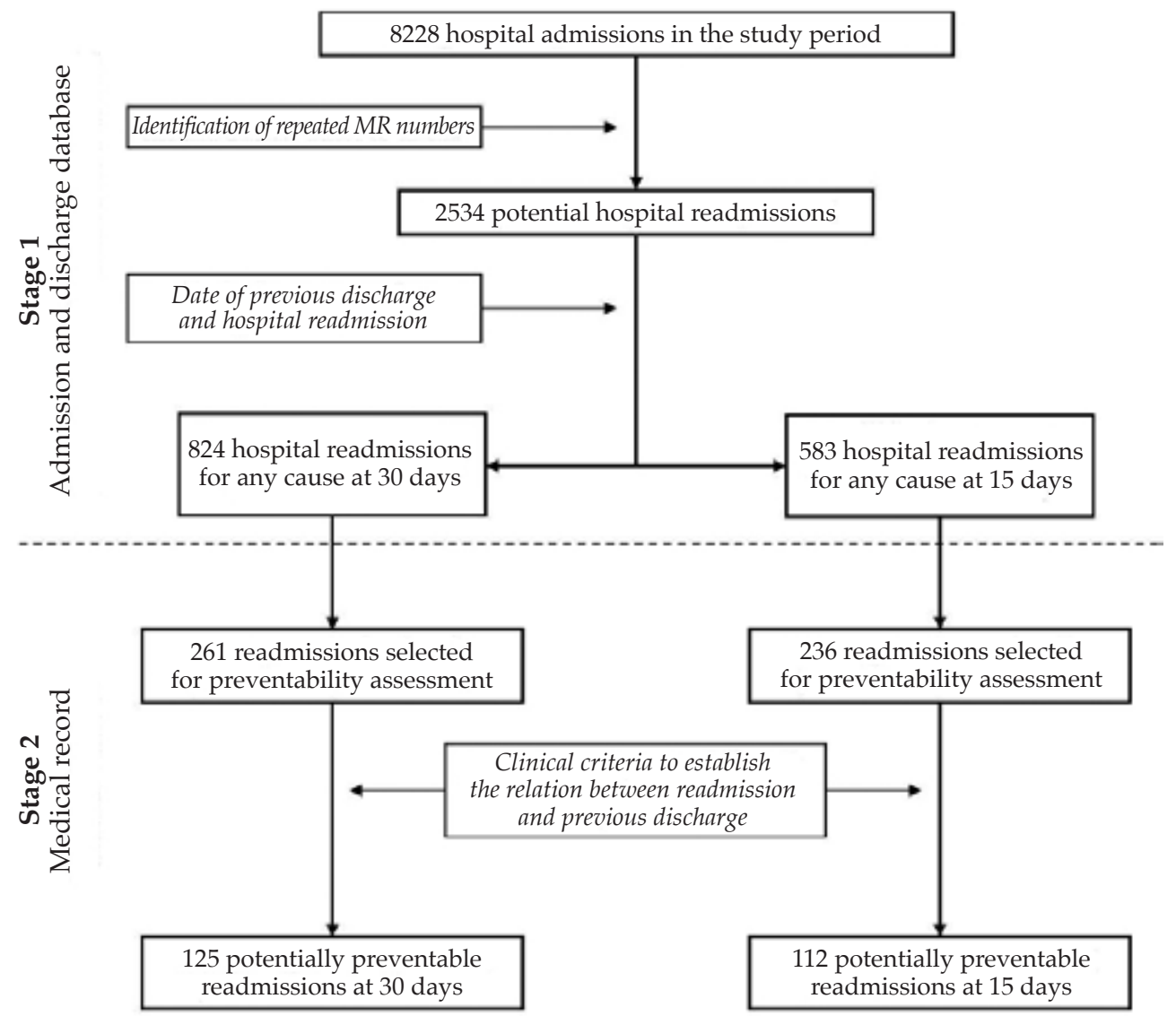


cause at 15 and 30 days. Patients with multiple admissions were selected by identifying repeated medical record numbers. Each repetition was assessed to identify whether it was a hospital readmission based on the criteria mentioned above. This way, the prevalence of HR for any cause at 15 and 30 days was estimated.

For the second stage, the prevalence of preventable HR was estimated using the criteria by Goldfield et al., ${ }^{7}$ based on the data obtained from the medical records. To this end, the sample size was re-estimated considering the prevalence of preventable readmissions described by PérezMoreno et al., ${ }^{2}$ and the number of readmissions identified in the first stage of the study. Lastly, the characteristics of potentially preventable HR at 15 and 30 days were compared.

Sample size: Based on a prevalence of HR of $4.1 \% \pm 0.5 \%^{2}$ and a $95 \%$ confidence level, the sample size was estimated at 5866 patients. However, all data for 2018 were analyzed to prevent any potential seasonal bias.

To estimate the rate of preventable readmissions, a prevalence of $42 \% \pm 5 \%,{ }^{2}$ a $95 \%$ confidence level, and a population of 583 readmissions at 15 days and 824 readmissions at 30 days were considered. This way, a sample of 236 readmissions was calculated to estimate the rate of preventable readmissions at 15 days and of 261 readmissions to estimate the rate of preventable readmissions at 30 days. The sample was selected by simple random sampling.

Statistical considerations: Categorical variables were described as percentages with their corresponding $95 \%$ confidence intervals (CIs). Continuous variables were expressed as median and range because distribution was not normal. To compare categorical variables, the $\chi^{2}$ test was used, whereas the Mann-Whitney U test was used to compare continuous ones. In all cases, a value of $p<0.05$ was considered significant.

Ethical considerations: The project was approved by the hospital's Ethics and Research Committee and registered at the Public Registry of Health Research of the Government of the Autonomous City of Buenos Aires (no. 261/19).

\section{RESULTS}

Prevalence of readmissions: During the study period, 8228 hospital admissions were recorded. No admissions met the exclusion criteria.

Among admissions, 824 were readmissions in the 30 days after the previous discharge, with a rate of readmissions for any cause of $10 \%(95 \% \mathrm{CI}$ : 9.4-10.7). A total of 583 readmissions occurred in the 15 days after the previous discharge, with a rate of readmissions for any cause of $7.1 \%$ (95\% CI: 6.6-7.7). Both admissions and readmissions showed a seasonal distribution; from May to July, the number of admissions and readmissions was higher (Figure 2).

Proportion of potentially preventable readmissions: Out of 261 readmissions at 30 days, $47.9 \%$ (95 \% CI: 41.2-53.9) were potentially preventable. Out of 236 readmissions at 15 days, $47.5 \%$ (95 \% CI: 41.2-53.8) were potentially

FIGURE 2. Number of admissions and readmissions for any cause by month

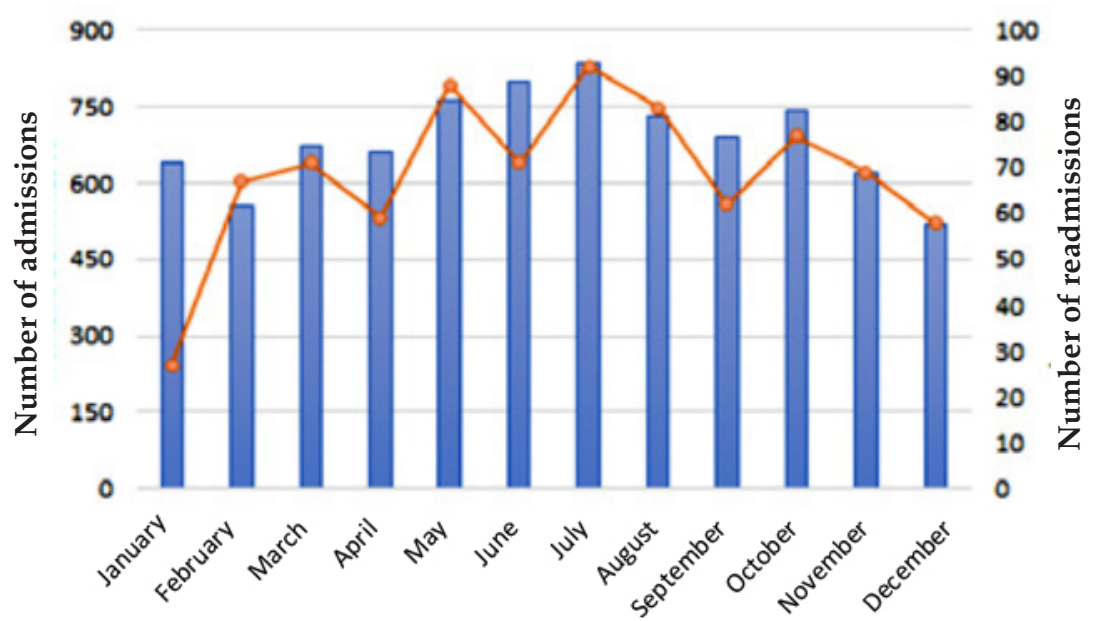

The bars represent the left $\mathrm{Y}$ axis. The lines represent the right $\mathrm{Y}$ axis. 
preventable. No statistically significant differences were observed between both groups. Characteristics of potentially preventable readmissions: In the group of readmissions at 15 days, $58.9 \%$ were due to recurrence of the same disease that caused the previous admission; $50.9 \%$ due to a complication associated with the previous admission; and $56.3 \%$ due to an acute decompensation of a chronic condition (patients may have more than 1 reason for readmission). In the group of readmissions at 30 days, $48.8 \%$ were due to recurrence of the same reason that led to the previous admission; $52 \%$ due to a complication associated with the previous admission; and $54.4 \%$ due to an acute decompensation of a chronic condition. No difference described here was statistically significant (Table 1).

The median age of patients with a potentially preventable readmission at 30 days was 3.9 years, whereas in the group at 15 days, it was 5.3 years. Also, $71.2 \%$ of patients with readmission at 30 days and $64.3 \%$ of those with readmission at 15 days had public health insurance exclusively. In addition, $73.2 \%$ of those in the group with readmission at 15 days and $71.2 \%$ of those in the group with readmission at 30 days had a chronic condition. No statistically significant differences were observed between both groups in relation to these characteristics.

\section{DISCUSSION}

This study describes the prevalence of readmissions for any cause at 15 and 30 days and the proportion of potentially preventable readmissions. The rate of readmissions for any cause at 30 days was $10 \%$, similar to that reported by Auger in Cincinnati $(11.3 \%),{ }^{8}$ but higher than that informed by Pérez-Moreno in Madrid $(4.1 \%)^{2}$ and by Toomey in Boston $(6.5 \%){ }^{9}$ Such differences may be explained because readmissions due to cancer or surgery were excluded completely by Pérez-Moreno and partially by Toomey. This type of conditions are a major cause for readmissions, ${ }^{4}$ which demonstrates that excluding certain causes for readmission may reduce its rate. It is worth wondering whether excluding these causes reduces the representativeness of this indicator. In the case of readmissions at 15 days, the experience is lower because the most common rate of readmissions is at 30 days. In this study, the rate of readmissions for any cause at 15 days

TABLE 1. Comparison of the rate and characteristics of potentially preventable readmissions

\begin{tabular}{|c|c|c|c|c|}
\hline & 15 days & 30 days & OR $(95 \%$ CI $)$ & $p^{*}$ \\
\hline Potentially preventable readmissions, \% (95\% CI) & $47.5(41.2-53.8)$ & $47.9(41.2-53.9)$ & $0.98(0.70-1.40)$ & 0.99 \\
\hline Characteristics of potentially preventable admissions & $\mathbf{n}=\mathbf{1 1 2}$ & $\mathbf{n}=\mathbf{1 2 5}$ & & \\
\hline Decimal age, median (range) & $5.3(0.1-18.7)$ & $3.9(0.1-18.5)$ & & 0.65 \\
\hline Presence of chronic condition, $\mathrm{n}(\%)$ & $82(73.2)$ & $89(71.2)$ & $1.11(0.63-1.95)$ & 0.84 \\
\hline \multicolumn{5}{|l|}{ Diagnosis based on ICD-10 chapters } \\
\hline Neoplasms & $27(24.1)$ & $28(22.4)$ & $1.10(0.60-2.01)$ & 0.88 \\
\hline Respiratory system & $17(15.2)$ & $23(18.4)$ & $0.73(0.37-1.44)$ & 0.46 \\
\hline Congenital malformations & $14(12.5)$ & $12(9.6)$ & $1.35(0.59-3.05)$ & 0.61 \\
\hline Digestive system & $5(4.5)$ & $2(1.6)$ & $0.93(0.28-3.12)$ & 0.90 \\
\hline Mental disorders & $5(4.5)$ & $6(4.8)$ & $0.93(0.28-3.12)$ & 0.90 \\
\hline Nervous system & $4(3.6)$ & $6(4.8)$ & $0.73(0.20-2.67)$ & 0.88 \\
\hline Endocrine, nutritional and metabolic diseases & $4(3.6)$ & $3(2.4)$ & $1.51(0.33-6.88)$ & 0.88 \\
\hline Other & $5(4.5)$ & $9(7.2)$ & $0.60(0.20-1.85)$ & 0.54 \\
\hline Public health insurance exclusively, n (\%) & $72(64.3)$ & $89(71.2)$ & $0.73(0.42-1.26)$ & 0.32 \\
\hline Length of stay in days during readmission, median (range) & $6(1-59)$ & $5(1-143)$ & & 0.44 \\
\hline \multicolumn{5}{|l|}{ Reason for readmission } \\
\hline Recurrence of the same reason as previous admission, $\mathrm{n}(\%)$ & $66(58.9)$ & $61(48.8)$ & $1.51(0.90-2.52)$ & 0.15 \\
\hline Decompensation of chronic condition, $\mathrm{n}(\%)$ & $63(56.3)$ & $68(54.4)$ & $1.10(0.65-1.80)$ & 0.88 \\
\hline Complication related to previous admission, $\mathrm{n}(\%)$ & $57(50.9)$ & $65(52.0)$ & $0.96(0.57-1.59)$ & 0.97 \\
\hline
\end{tabular}

* In all cases, a value of $p<0.05$ was considered significant. Categorical variables were compared using the $\chi^{2}$ test.

Continuous variables were compared using the Mann-Whitney U test.

CI: confidence interval; OR: odds ratio; ICD-10: International Classification of Diseases, $10^{\text {th }}$ revision. 
$(7.1 \%)$ was similar to that described by Gay $(8.4 \%)$ at a hospital of Vanderbilt. ${ }^{10}$ In that study, the authors included all readmissions, without excluding any cause.

In this study, the rate of potentially preventable readmissions was also analyzed and was $47.9 \%$ at 30 days and $47.5 \%$ at 15 days. Pérez-Moreno described a similar rate $(46.4 \%))^{2}$ This study defined preventable readmissions as those occurring in the 15 days after discharge and for the same cause as the previous admission. Toomey estimated a lower rate of potentially preventable readmissions $(29.5 \%) .{ }^{9}$ The authors defined potentially preventable readmissions based on the review of medical records and interviews with parents and/or caregivers. The information gathered during the interviews changed the classification of preventable to non-preventable in $11.8 \%$ of cases. The differences observed in the rate of potentially preventable readmissions may be related to the methods used to categorize them. The use of administrative information, clinical data or parents' feedback shows varying results, depending on how they are used or combined. 5,6 Another characteristic of patients with potentially preventable readmissions that is worth noting is the high prevalence of chronic conditions. In our study, the prevalence of chronic conditions was $71.2 \%$ and $73.2 \%$ for readmissions at 30 and 15 days, respectively. Such rate is similar to those reported in other studies, from $63 \%$ to $79 \% .2,9,10$ It makes sense to believe that the population of patients with chronic diseases is more prone to be hospitalized, either due to a decompensation or a complication associated with their underlying condition. In any case, these values demonstrate the relevance of this population, and any strategy targeted at reducing readmissions should focus especially on it.

Most readmissions assessed here had public health insurance exclusively. Such proportion is higher than that observed in studies conducted in other countries. ${ }^{3}$ An exclusive public health insurance may be considered an indirect marker of social vulnerability in our setting and implies, almost inevitably, that the parents of patients lack a formal employment. A greater social vulnerability may explain, in part, the higher rate of readmissions described in this study compared to other authors. ${ }^{2,9}$ Socioeconomic factors are associated with the variability in the rates of pediatric readmissions between hospitals. ${ }^{4}$

In addition to patient characteristics, pediatric readmissions may be affected by environmental aspects. Throughout the year, readmissions showed a seasonal distribution and the months with respiratory virus circulation prevailed. Pérez-Moreno observed a similar distribution in the Northern Hemisphere. ${ }^{2}$ This may be explained by 2 factors. First of all, the direct effect of respiratory tract infections. Patients who were recently discharged for any cause are more likely to develop a respiratory tract infection resulting in a readmission. Secondly, the pressure caused by the high levels of bed occupancy on facilities. Such pressure may result in patients being discharged too early and, therefore, potentially preventable readmissions.

In spite of the efforts made to reduce readmissions, the rates remain unchanged. ${ }^{6}$ One of the factors that may account for this is that the rate of readmissions is estimated at 30 days with readmissions for any cause. The strategies implemented to reduce readmissions may be effective in certain patient subgroups, but not in all of them, and small achievements may not be reflected in the overall rate. It is worth noting that, according to this study, the populations of patients with readmissions for any cause at 15 and 30 days are similar. Therefore, it may be possible to implement a single strategy aimed at reducing readmissions in these patients.

One of the potential limitations of this study is that the analysis was performed exclusively in relation to readmissions at a tertiary care children's hospital, with potential biases in relation to pediatric care in a general hospital. In addition, potential readmissions with a previous admission in a different facility were not taken into account. However, readmissions to the same facility may be an indicator of a better health care quality ${ }^{11}$ because pediatric patients usually meet their hospital care needs at a single facility. ${ }^{4}$ In relation to the transition to home care, there was no information available about outpatient follow-up, treatment compliance, and access to medication after discharge (variables that may affect the rate of readmissions). Notwithstanding this, all the population admitted to the hospital was included, without excluding cancer or surgery patients, so the results described here account for the entire hospital population. In addition, the assessment covered a complete year to avoid any seasonal bias typical of pediatric diseases.

The primary outcome variable is considered a strength of this study. An objective definition was used to classify readmissions as potentially preventable, and each medical record was 
reviewed to establish the characteristics of this population. This is an easily reproducible methodology and provides the study with external and methodological validity.

Lastly, it is worth noting that the interest in the rate of readmissions is rising worldwide. In the United States, much of such interest is supported by the financial penalties that may be imposed on facilities for this reason. 8,12 The Argentine health system has not prioritized the rate of $\mathrm{HR}$ as a measure of quality and, therefore, no incentive initiative to reduce HR has been put into place. To study such phenomenon for the first time, it is critical to know the rate of readmissions and their characteristics at different facilities. Since it depends on many variables, results may be specific to each facility or comparable only between similar facilities. This study is a starting point to analyze a multifactorial phenomenon with negative effects on patients, their families, ${ }^{13,14}$ and the health system. ${ }^{3}$

\section{CONCLUSION}

The rate of HR for any cause was $10 \%$ at 30 days of discharge and $7.1 \%$ at 15 days of discharge. The proportion of potentially preventable readmissions was $47.9 \%$ at 30 days and $47.5 \%$ at 15 days. No significant differences were observed in the characteristics of potentially preventable readmissions at 15 days and 30 days in relation to health insurance and presence of a chronic condition.

\section{REFERENCES}

1. Zhou H, Della P, Roberts P, Porter P, et al. A 5-year retrospective cohort study of unplanned readmissions in an Australian tertiary paediatric hospital. Aust Health Rev. 2019; 43(6):662-71.

2. Pérez-Moreno J, Leal-Barceló AM, Márquez Isidro E, Toledo-Del Castillo B, et al. Detección de factores de riesgo de reingreso prevenible en la hospitalización pediátrica. An Pediatr (Barc). 2019; 91(6):365-70.

3. Markham JL, Hall M, Gay JC, Bettenhausen JL, et al. Length of Stay and Cost of Pediatric Readmissions. Pediatrics. 2018; 141(4):e20172934.

4. BerryJG, Toomey SL, Zaslavsky AM, Jha AK, et al.Pediatric readmission prevalence and variability across hospitals. JAMA. 2013; 309(4):372-80.

5. Auger KA, Ponti-Zins MC, Statile AM, Wesselkamper K, et al. Performance of Pediatric Readmission Measures. J Hosp Med. 2020; 15(12):723-6.

6. Auger KA, Harris JM, Gay JC, Teufel R, et al. Progress (?) Toward Reducing Pediatric Readmissions. J Hosp Med. 2019; 14(10):618-21.

7. Goldfield NI, McCullough EC, Hughes JS, Tang AM, et al. Identifying potentially preventable readmissions. Health Care Financ Rev. 2008; 30(1):75-91.

8. Auger KA, Teufel RJ 2nd, Harris JM 2nd, Gay JC, et al. Children's Hospital Characteristics and Readmission Metrics. Pediatrics. 2017; 139(2):e20161720.

9. Toomey SL, Peltz A, Loren S, Tracy M, et al. Potentially Preventable 30-Day Hospital Readmissions at a Children's Hospital. Pediatrics. 2016; 138(2):e20154182.

10. Gay JC, Hain PD, Grantham JA, Saville BR. Epidemiology of 15-Day Readmissions to a Children's Hospital. Pediatrics. 2011; 127(6):e1505-12.

11. Khan A, Nakamura MM, Zaslavsky AM, Jang J, et al. SameHospital Readmission Rates as a Measure of Pediatric Quality of Care. JAMA Pediatr. 2015; 169(10):905-12.

12. MannDL, TomaselliGF. The AffordableCare Act's Hospital Readmissions Reduction Program: Has the Biology Had Time to Catch Up With the Regulation? JACC Basic Transl Sci. 2016; 1(7):687-9.

13. Allshouse C, Comeau M, Rodgers R, Wells N. Families of Children With Medical Complexity: A View From the Front Lines. Pediatrics. 2018; 141(Suppl 3):S195-201.

14. Leary JC, Krcmar R, Yoon GH, Freund KM, et al. Parent Perspectives During Hospital Readmissions for Children With Medical Complexity: A Qualitative Study. Hosp Pediatr. 2020; 10(3):222-9. 